(1)

CrossMark

\title{
HFE gene variants and iron-induced oxygen radical generation in idiopathic pulmonary fibrosis
}

\author{
Federica Sangiuolo ${ }^{1,11}$, Ermanno Puxeddu ${ }^{1,11}$, Gabriella Pezzuto², \\ Francesco Cavalli ${ }^{3}$, Giuliana Longo ${ }^{1}$, Alessia Comandini ${ }^{2}$, Donato Di Pierro ${ }^{4}$, \\ Marco Pallante ${ }^{1}$, Gianluigi Sergiacomi ${ }^{5}$, Giovanni Simonetti ${ }^{5}$, Maurizio Zompatori ${ }^{6}$, \\ Augusto Orlandi ${ }^{7}$, Andrea Magrini ${ }^{1}$, Massimo Amicosante ${ }^{1}$, Francesca Mariani $^{8}$, \\ Monica Losi ${ }^{2}$, Daniela Fraboni ${ }^{9}$, Alberto Bisetti ${ }^{10,+}$ and Cesare Saltini ${ }^{1,2,3}$

\begin{abstract}
Affiliations: ${ }^{1}$ Dept of Biomedicine and Prevention, University of Roma Tor Vergata, Rome, Italy. ${ }^{2}$ Division of Respiratory Diseases, University Hospital Tor Vergata, Rome, Italy. ${ }^{3}$ Postgraduate School in Respiratory Diseases, University of Roma Tor Vergata, Rome, Italy. ${ }^{4}$ Dept of Clinical Science and Translation Medicine, University of Roma Tor Vergata, Rome, Italy. ${ }^{5}$ ept of Diagnostic Imaging, Molecular Imaging, Interventional Radiology and Radiation Therapy, University Hospital Tor Vergata, Rome, Italy. ${ }^{6}$ Dept of Diagnostic Imaging, Molecular Imaging, Interventional Radiology and Radiotherapy, University of Bologna, Bologna, Italy. ${ }^{7}$ Dept of Anatomic Pathology, University of Roma Tor Vergata, Rome, Italy. ${ }^{8}$ Institute Cell Biology and Neurobiology, National Research Council, Monterotondo Scalo, Italy. ${ }^{9}$ Dept of Laboratory Medicine, University Hospital Tor Vergata, Rome, Italy. ${ }^{10}$ University of Roma La Sapienza, Rome, Italy. ${ }^{11}$ Both authors contributed equally.
\end{abstract}

Correspondence: Cesare Saltini, University of Rome Tor Vergata, Dept of Biomedicine and Prevention, Via Montpellier 1, 00133 Rome, Italy. E-mail: saltiniamed.uniroma2.it

ABSTRACT In idiopathic pulmonary fibrosis (IPF), lung accumulation of excessive extracellular iron and macrophage haemosiderin may suggest disordered iron homeostasis leading to recurring microscopic injury and fibrosing damage.

The current study population comprised 89 consistent IPF patients and 107 controls. 54 patients and 11 controls underwent bronchoalveolar lavage (BAL). Haemosiderin was assessed by Perls' stain, BAL fluid malondialdehyde (MDA) by high-performance liquid chromatography, BAL cell iron-dependent oxygen radical generation by fluorimetry and the frequency of hereditary haemochromatosis HFE gene variants by reverse dot blot hybridisation.

Macrophage haemosiderin, BAL fluid MDA and BAL cell unstimulated iron-dependent oxygen radical generation were all significantly increased above controls $(\mathrm{p}<0.05)$. The frequency of $\mathrm{C} 282 \mathrm{Y}, \mathrm{S} 65 \mathrm{C}$ and H63D HFE allelic variants was markedly higher in IPF compared with controls (40.4\% versus $22.4 \%$, OR 2.35, $\mathrm{p}=0.008$ ) and was associated with higher iron-dependent oxygen radical generation (HFE variant 107.4 \pm 56.0 , HFE wild type (wt) $59.4 \pm 36.4$ and controls $16.7 \pm 11.8$ fluorescence units per $10^{5}$ BAL cells; $\mathrm{p}=0.028$ HFE variant versus $\mathrm{HFE}$ wt, $\mathrm{p}=0.006$ HFE wt versus controls).

The data suggest iron dysregulation associated with HFE allelic variants may play an important role in increasing susceptibility to environmental exposures, leading to recurring injury and fibrosis in IPF.

@ERSpublications

HFE gene variants that cause iron-related fibrosing diseases implicate lung iron dysregulation in IPF pathogenesis http://ow.ly/DRnJK 


\section{Introduction}

Idiopathic pulmonary fibrosis (IPF) is the most common and rapidly fatal among idiopathic interstitial pneumonias, a group of interstitial disorders of unknown origin limited to the lung [1]. IPF is characterised by the pattern of usual interstitial pneumonia (UIP), with the appearance of areas of reticular abnormalities and honeycomb cysts alternating with areas of less-affected parenchyma on high-resolution computed tomography (HRCT) of the chest and, on surgical lung biopsy, with focal fibroblast proliferation resulting from microscopic foci of acute lung injury of unknown nature [2,3]. Bronchoalveolar lavage (BAL) studies in IPF have shown increased alveolar macrophage and polymorphonuclear leukocyte (PMN) numbers and spontaneous reactive oxygen species (ROS) release [4-6]. Experimental pulmonary fibrosis models, including bleomycin and silica, indicate $\mathrm{OH}$-radical generation in the Fenton reaction as a likely cause of damage, hence as a trigger of the ensuing fibrogenic cascade [7-9]. Iron accumulation has been observed in IPF patients with associated pulmonary hypertension [10]. Using bronchiolo-alveolar samples recovered by BAL, we found increased concentrations of extracellular alveolar iron and alveolar macrophage haemosiderin in most IPF patients [11], an observation suggesting that iron-dependent ROS generation, a key feature of iron overload fibrosing diseases [12], could play a role in IPF as well.

The regulation of iron import into the cell is normally controlled by the iron-induced HFE (haemochromatosis) protein, which, by binding to the transferrin receptor, blocks transferrin-bound iron import. Iron export is controlled by ferroportin, an iron exporter present on the surface of absorptive enterocytes, macrophages and hepatocytes that releases iron into the plasma. In type 1 hereditary haemochromatosis (OMIM (Online Mendelian Inheritance in Man, http://omim.org) \#235200), a common autosomic recessive disorder of iron homeostasis, the regulation of iron import into the cell is altered by HFE gene mutations, which are thought to abolish the ability of the HFE protein to bind to the transferrin receptor and limit iron import, hence leading to iron overload and endoplasmic reticulum stress. Conversely, type 4 hereditary haemochromatosis (OMIM \#606069), an uncommon autosomal dominant disorder more recently defined as ferroportin disease, is characterised by alteration of iron release from the cell caused by mutations of the ferroportin gene [13-16].

Interestingly, the role and physiological consequences of the C282T and the H63D HFE gene mutations have been elucidated in a knock-in murine model of hereditary haemochromatosis generating C294Y homozygous, H67D homozygous and H67D/C294Y compound heterozygous knock-in mice (corresponding to $\mathrm{C} 282 \mathrm{Y}$ and $\mathrm{H} 63 \mathrm{D}$ in humans). In all conditions, liver iron overload was shown, with a decreasing severity from homozygous C294Y/C294Y to compound heterozygous C294Y/H67D down to heterozygous $\mathrm{H} 67 \mathrm{D}$, indicating that the $\mathrm{H} 67 \mathrm{D}$ mutation in the mouse, corresponding to the H63D in the human, may contribute to a haemochromatosis phenotype by leading to partial loss of HFE function [17]. In humans, both variants have been shown to determine an increase in blood iron and transferrin saturation [18]. C282Y and H63D are common allelic variants of HFE, with allele frequencies of $3.8 \pm 0.7 \%$ and $13.6 \pm 1.3 \%$, respectively, in Europe, and lower frequencies for $\mathrm{C} 282 \mathrm{Y}$ in southern Europe and Italy [19]. Importantly, they have been reported, albeit not univocally, to confer greater susceptibility to liver iron overload and cirrhosis upon heterozygous carriers exposed to chronic inflammation, such as in viral hepatitis or alcohol-related liver disease $[20,21]$. With this as a background, and with the knowledge that a number of exposures have been implicated as possible risk factors for IPF [22, 23], we hypothesised a role as susceptibility factors to HFE allelic variants, due to their potential for dysregulated iron accumulation and exaggerated generation of oxygen radicals.

\section{Methods}

\section{Control and IPF populations}

The control population comprised 107 healthy subjects, $98.1 \%$ of whom were born in central-southern Italy. Their mean \pm SD age was $54.3 \pm 4.3$ years. $86.0 \%$ were males and $61.7 \%$ were current or past smokers. A subpopulation of 11 subjects (aged $50.4 \pm 6.6$ years, five males, five current smokers) was enrolled under an interventional study protocol (approved by the Fondazione Policlinico Tor Vergata (FPTV) Independent Ethics Committee) to undergo fibreoptic BAL. All gave informed consent (approved by the FPTV Independent Ethics Committee) to donate a blood sample for genetic studies.

Support statement: The work was supported by a grant from FILAS/Regione Lazio (Italy) to Research and Drug Development (ReDD) Inc., Rome, Italy (a University of Roma Tor Vergata spin-off with which M. Amicosante, F. Mariani and C. Saltini are associated) and the University of Roma Tor Vergata, Rome, Italy; by an unrestricted research grant from the "Associazione Italiana Malattie Interstiziali del Polmone", Rome, Italy (awarded to M. Losi); by an unrestricted grant from Boehringer Ingelheim; and by a fellowship in memory of Virgilio Guzzini, from the Guzzini family, Recanati, Italy (awarded to M. Amicosante at the inception of the study).

Conflict of interest: Disclosures can be found alongside the online version of this article at erj.ersjournals.com 
The IPF patient population comprised 89 individuals, $95.5 \%$ of whom were born in central-southern Italy. Their mean \pm SD age was $68.8 \pm 7.6$ years. $75.3 \%$ were males and $75.3 \%$ were current or past smokers (mean \pm SD $32.2 \pm 21.6$ pack-years and $17.5 \pm 11.8$ years after smoking cessation) with no known exposure to fibrogenic dusts. A subgroup of 54 patients aged $68.4 \pm 7.4$ years $(79.6 \%$ males, $75.9 \%$ ever-smokers, $32.8 \pm 24.3$ pack-years) was evaluated by BAL as part of the standard diagnostic work-up. They were not different from the whole patient population for any of the demographic parameters ( $p>0.05$, all comparisons). All gave informed consent (approved by the FPTV Independent Ethics Committee) to the use of a portion of their biological samples, remaining after diagnostic tests, for research use, including genetic testing.

The diagnosis of IPF was established, or revised for those enrolled in the year 2011 and before, according to the recommendations of the 2011 official American Thoracic Society/European Respiratory Society/ Japanese Respiratory Society/Asociación Latinoamericana de Tórax statement on IPF [2]. In particular, multidisciplinary discussions were carried out by three pulmonary disease specialists (Ermanno Puxeddu, Gabriella Pezzuto and Cesare Saltini), three radiologists (Gianluigi Sergiacomi, Giovanni Simonetti and Maurizio Zompatori) and a pathologist (Augusto Orlandi) following the analytical evaluation of HRCT and histological patterns outlined by the official statement [2]. The diagnosis of IPF was based upon the presence of a confident UIP pattern on HRCT $(n=79)$ or a possible/inconsistent UIP HRCT pattern in combination with UIP-consistent histology at surgical lung biopsy $(n=10)$.

\section{Pulmonary function testing}

Pulmonary function testing included forced vital capacity (FVC) and forced expiratory volume in $1 \mathrm{~s}$ $\left(\mathrm{FEV}_{1}\right)$ and, in IPF patients, total lung capacity, residual volume and diffusing capacity of the lung for carbon monoxide (DLCO). Tests were performed with a Jaeger MasterScreen PFT analyser unit (Jaeger, Würzburg, Germany).

\section{Serum iron measurement}

Serum iron measurements, including iron, transferrin and ferritin, were performed using the Siemens Dimension Vista System Flex reagent cartridge (Siemens Healthcare Diagnostics Products, Duisburg Rheinhausen, Germany).

\section{$B A L$}

BAL was performed using a flexible fibreoptic bronchoscope, Olympus BF 1T 180 (Olympus, Hamburg, Germany), according to American Thoracic Society guidelines [24]. Differential cell counts were performed using a Cytospin Shandon 4 (Thermo Scientific, Waltham, MA, USA) (table 1).

\section{Alveolar macrophage haemosiderin}

Haemosiderin-laden alveolar macrophages were determined by means of the Perls' iron stain (Bio-Optica, Milan, Italy), according to GolDE et al. [25].

\section{Malondialdehyde}

Malondialdehyde (MDA) quantification in BAL fluids was performed as described by LAZZARINO et al. [26]. The high-performance liquid chromatography (HPLC) apparatus consisted of a Surveyor LC Pump (Thermo Finnigan Italia SpA, Rodano, Milan, Italy) connected to a Surveyor PDA (photo diode array) Detector (Thermo Finnigan Italia SpA) with a wavelength range of 200-300 nm. Data were acquired and analysed with the ChromQuest software (Thermo Scientific). Areas, retention time and absorbance spectra of the MDA peak at $267 \mathrm{~nm}$ were compared with that of freshly prepared ultrapure standard.

$30 \mathrm{~mL}$ of BAL was lyophilised and resuspended in a final volume of $2 \mathrm{~mL}$. $20 \mu \mathrm{L}$ of ice cold $20 \%$ perchloric acid was added and the sample centrifuged at $20600 \mathrm{~g}$ for $10 \mathrm{~min}$ at $4^{\circ} \mathrm{C}$, then neutralised with $5 \mathrm{M}$ potassium bicarbonate on ice and filtered through a $0.45-\mu \mathrm{m}$ Millipore HV filter (Millipore, Cork, Ireland) before injection into HPLC apparatus.

Free MDA was obtained from malonaldehyde bis (diethyl acetal) (Merck, Darmstadt, Germany), as described by Esterbauer et al. [27]. Tetrabutylammonium hydroxide, 55\% aqueous solution, was purchased from Novachimica (Milan, Italy).

\section{Iron-dependent ROS generation}

Generation of iron-dependent ROS was estimated as the amount of ROS-induced 5-(and-6)-chloromethyl2',7'-dichlorodihydrofluorescein diacetate acetyl ester (CM-H2DCFDA) (Invitrogen, Molecular Probes, Carlsbad, CA, USA) fluorescence generated by fresh, unstimulated BAL cells that could be inhibited by the iron chelator deferoxamine (DFO; $100 \mu \mathrm{M})$ using a modification of the procedure described by BASS et al. 
[28]. Briefly, $1 \times 10^{5}$ unseparated BAL cells, washed twice in PBS, were resuspended in RPMI without phenol red (Sigma Aldrich, St Louis, MO, USA) and incubated in the dark in the presence/absence of DFO (Sigma Aldrich) at a final concentration of $100 \mu \mathrm{M}$ for $1 \mathrm{~h}\left(37^{\circ} \mathrm{C}, 5 \% \mathrm{CO}_{2}\right)$. The fluorochrome CM-H2DCFDA was added thereafter at a final concentration of $5 \mathrm{mM}$ and fluorescence was measured, after an incubation of $1 \mathrm{~h}$, with an LS50B Luminescence Spectrometer (Perkin Elmer, Waltham, MA, USA) at excitation and emission wavelengths of 485 and $535 \mathrm{~nm}$, respectively, with a 2.5-nm window. Auto-fluorescent beads (FluoSpheres Sulfate Microspheres; Invitrogen, Molecular Probes) were used as a sensitivity control. Iron-dependent ROS generation was expressed as the amount of DFO-inhibited CM-H2DCFDA fluorescence intensity units (DCFDA/DFO-FU per $10^{5}$ BAL cells).

\section{Mutation analysis}

Mutation analysis of the hereditary haemochromatosis genes was carried out on DNA isolated from peripheral blood using EZ1_DNA Blood 200 (Qiagen GmbH, Hilden, Germany), or from BAL or paraffin-embedded tissues from surgical lung biopsy using EZ1_DNA Investigator Kit (Qiagen GmbH), following the manufacturer's instructions. The sequences of interest of the HFE gene were amplified in multiplex and the biotinylated amplicons were hybridised to allele-specific probes (reverse dot blot), as recommended by the manufacturer (Nuclear Laser Medicine, Milan, Italy). Biotinylated hybrids were successively revealed using streptavidin conjugated to alkaline phosphatase and coloured substrate. 11 specific mutations within the HFE gene were simultaneously screened (V53M, V59M, H63D, S65C, Q127H, E168Q, E168X, W169X, C282Y, Q283P and P160 del C), in addition to four mutations of the transferrin 2 gene (Y250X, E60X, M172 K and AVA Q 594-597 del) and two mutations of the ferroportin gene $(\mathrm{N} 144 \mathrm{H}$ and V162 del).

\section{Statistical analysis}

Study population data were collected and analysed according to the STREGA (STrengthening the REporting of Genetic Association studies) recommendations [29]. Data are presented as mean \pm SD or percentages, as most appropriate. Comparisons between groups were evaluated by Mann-Whitney U-test. Comparisons between genotype frequencies were evaluated by univariate and multivariate binary logistic regression. Values of $\mathrm{p}<0.05$ were considered significant. The odds ratio was calculated as the ratio of gene polymorphism frequency in IPF patients with respect to healthy controls. GraphPad Prism version 5.0 (Graphpad Software, San Diego, CA, USA) and SPSS Software version v20 (IBM, Armonk, NY, USA) were used for statistical analyses and graphs.

\section{Results}

The IPF and control populations were not significantly different with regard to geographical origin, sex and smoking history, while IPF patients were on average about 15 years older than healthy controls (see Methods section). All healthy controls had normal pulmonary function (FVC $103.9 \pm 12.7 \%$ predicted; FEV1 $99.8 \pm 12.7 \%$ pred). As expected, IPF study patients had low FVC (75.5 $\pm 23.4 \%$ pred) with normal FEV1 $(80.5 \pm 22.8 \%$ pred $)$ and low total lung capacity $(67.2 \pm 17.0 \%$ pred $)$ and DLCO $(42.1 \pm 17.7 \%$ pred $)$. On average, IPF study patients had blood iron profiles within normal limits (serum iron $82.0+32.2 \mathrm{mg} \cdot \mathrm{dL}^{-1}$, reference values 50-175 $\mathrm{mg} \cdot \mathrm{dL}^{-1}$; serum transferrin $243.1 \pm 41.7 \mathrm{mg} \cdot \mathrm{dL}^{-1}$, reference values $200-$ $\left.360 \mathrm{mg} \cdot \mathrm{dL}^{-1}\right)$.

Macrophage haemosiderin accumulation, as measured by the Golde score, was markedly increased in IPF-affected subjects compared with healthy subjects, smoking and nonsmoking combined (IPF ( $\mathrm{n}=54$ ) $54.0 \pm 43.4$ versus controls $(\mathrm{n}=11) 10.5 \pm 13.2 ; \mathrm{p}=0.018$ ) (table 2).

Similarly, the levels in BAL fluids of the lipid peroxidation adduct MDA were markedly elevated in IPF patients compared with controls (smoking and nonsmoking combined) (IPF ( $\mathrm{n}=31$ ) $0.0137 \pm 0.0061$ $\mathrm{nmol} \cdot \mathrm{mL}^{-1}$ versus controls $\left.(\mathrm{n}=11) 0.0011 \pm 0.0017 \mathrm{nmol} \cdot \mathrm{mL}^{-1} ; \mathrm{p}<0.005\right)$ (table 2 ).

Unstimulated, DFO-chelatable iron-dependent ROS-induced DCFDA fluorescence, generated by unseparated BAL cells from IPF patients ( $n=22,83.4 \pm 52.3$ DCFDA/DFO-FU per $10^{5}$ BAL cells) was significantly larger than in normal controls $\left(\mathrm{n}=9,16.7 \pm 11.8\right.$ DCFDA/DFO-FU per $10^{5}$ BAL cells; $\mathrm{p}<0.0001$ ) (fig. 1a).

Only three HFE allelic variants, H63D, S65C and C282Y, were identified, defining six different genotypes in the study population. The results of the multivariate binary logistic regression analysis of their frequencies in IPF patients compared with controls are summarised in table 3. The increased frequency of H63D in IPF patients was highly significant ( $p=0.004$; OR 2.60, 95\% CI 1.35-5.02). No transferrin receptor 2 or ferroportin gene variants were identified in the control or IPF patient populations. Overall, allelic variants were found in $36(40.4 \%)$ of the IPF-affected patients and in $24(22.4 \%)$ of the healthy controls ( $\mathrm{p}=0.008$; OR 2.35, 95\% CI 1.26-4.37). 
TABLE 1 Bronchoalveolar lavage cell recovery and differential counts

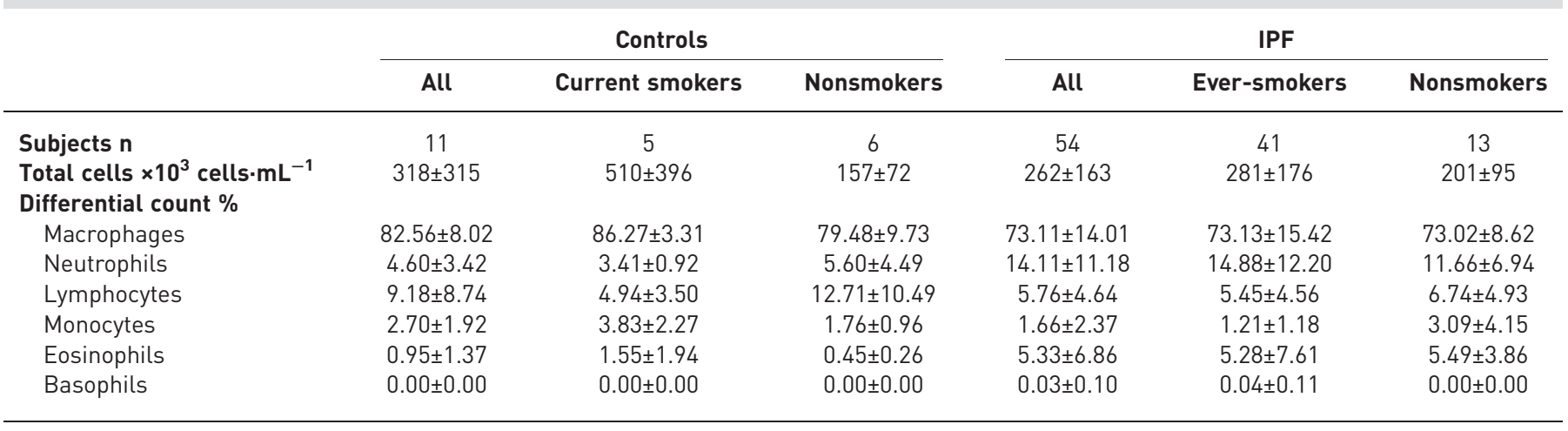

Data are presented as mean \pm SD, unless otherwise stated. IPF: idiopathic pulmonary fibrosis.

Stratification of IPF patients by HFE allelic variants showed that, while the intensity of haemosiderinic iron accumulation in macrophages and the concentration of MDA in BAL fluids were not different in wild type or in carriers of HFE variants $(\mathrm{p}>0.05)$, unstimulated, labile iron-dependent ROS generation was significantly greater in the IPF patients carrying HFE allelic variants $(n=11,107.4 \pm 56.0$ DCFDA/DFO-FU

\section{TABLE 2 Macrophage haemosiderin accumulation and malondialdehyde levels in} bronchoalveolar lavage fluid

\begin{tabular}{lccc} 
& Controls & IPF & p-value \\
\hline Haemosiderin intensity Golde score & $10.5 \pm 13.2$ & $54.0 \pm 43.4$ & $<0.02$ \\
Malondialdehyde $\mathbf{n m o l} \cdot \mathrm{mL}^{-1}$ & $0.0011 \pm 0.0017$ & $0.0137 \pm 0.0061$ & $<0.005$ \\
\hline
\end{tabular}

Data are presented as mean $\pm S D$, unless otherwise stated. IPF: idiopathic pulmonary fibrosis.
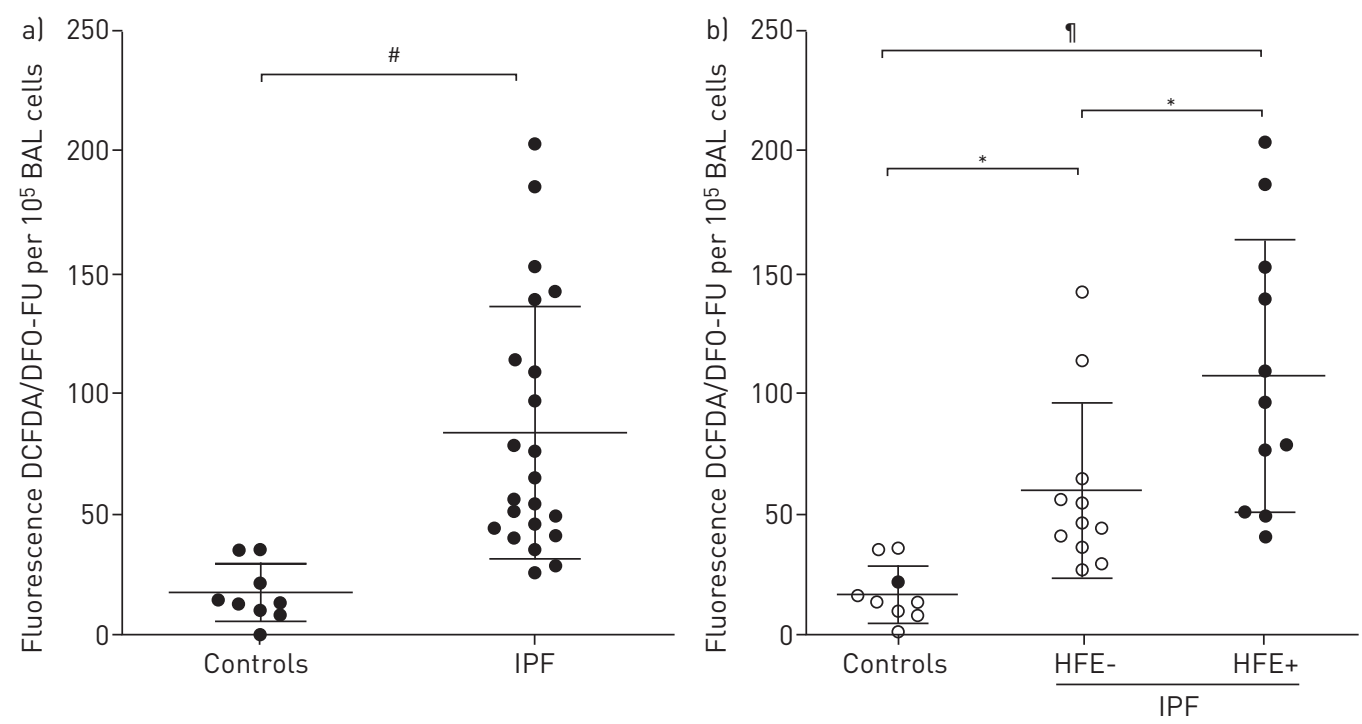

FIGURE 1 a) Amounts of iron-dependent, deferoxamine (DFO)-chelatable, reactive oxygen species-induced 5-(and-6)chloromethyl-2',7'-dichlorodihydrofluorescein diacetate acetyl ester (CM-H2DCFDA) generated by freshly recovered, unstimulated bronchoalveolar lavage (BAL) cells from healthy controls and from idiopathic pulmonary fibrosis (IPF) subjects. Fluorescence intensity units are DCFDA/DFO-FU per $10^{5}$ BAL cells. b) Data stratified by HFE (haemochromatosis) allelic variant expression. Controls and IPF-affected individuals (HFE-) homozygous for HFE wild-type sequence are shown as open circles, while controls and IPF-affected individuals (HFE+) expressing HFE variant alleles are shown as filled circles. Means and \pm 1 SD values are shown by horizontal lines and whiskers, respectively. ${ }^{*}$ : $\mathrm{p}<0.0001 ;{ }^{\text {ๆ }}$ : $\mathrm{p}<0.005 ;{ }^{*} \mathrm{p}<0.05$. 
TABLE 3 HFE (haemochromatosis) gene allelic variant analysis

\begin{tabular}{llcccc}
\multicolumn{2}{l}{ Genotype } & Controls $^{\#}$ & IPF $^{\Uparrow}$ & OR (95\% CI) & p-value \\
\hline WT & WT & $83(77.6)$ & $53(59.6)$ & $0.43(0.23-0.792)$ & 0.745 \\
H63D & WT & $19(17.8)$ & $32(36.0)$ & $2.60(1.35-5.02)$ & 0.004 \\
C282Y & WT & $4(3.7)$ & $1(1.1)$ & $0.30(0.32-2.67)$ & 0.368 \\
S65C & WT & $0(0.0)$ & $2(2.2)$ & NC & 0.077 \\
H63D & H63D & $0(0.0)$ & $1(1.1)$ & NC & 0.212 \\
H63D & C282Y & $1(0.9)$ & $0(0.0)$ & NC & 0.419 \\
\hline
\end{tabular}

Data are presented as $\mathrm{n}(\%)$, unless otherwise stated. IPF: idiopathic pulmonary fibrosis; WT: wild type; NC: not calculated. ${ }^{\#}: \mathrm{n}=107 ;{ }^{\text {ๆ }}: \mathrm{n}=89$.

per $10^{5}$ BAL cells) than in those showing wild-type HFE sequences ( $n=11,59.1 \pm 36.4$ DCFDA/DFO-FU per $10^{5}$ BAL cells; $\mathrm{p}=0.028$ ). However, both groups showed greater values than controls $\left(16.7 \pm 11.8\right.$ DCFDA/DFO-FU per $10^{5}$ BAL cells; $\mathrm{p}=0.003$ ) (fig. 1b). The control population comprised eight individuals carrying the HFE wild-type sequence and only one with the C282Y allelic variant.

\section{Discussion}

This study shows that in IPF the carriage of HFE allelic variants, primarily of the H63D variant, is associated with excessive, spontaneous, iron-dependent ROS generation by fresh, unstimulated bronchoalveolar cells. The lower respiratory tract is an iron-rich environment exposed to high oxygen concentrations where, normally, alveolar macrophages are capable of sequestering iron as ferritin-bound oxidised iron to keep it in an unreactive form. Increased levels of iron have been found in the lower respiratory tract of subjects with pulmonary idiopathic siderosis, where iron is entirely bound to ferritin to prevent excessive oxidative stress [30]. Increased levels of haemosiderin iron are also found in pulmonary veno-occlusive disease, where Prussian blue stained alveolar macrophages and type II pneumocytes are seen in the absence of frank fibrosis [31], and in diffuse alveolar damage, concomitant with moderate to severe alveolar haemorrhage [32]. Finally, in some transfusion-dependent thalassaemia patients, systemic iron overload has been associated with functional and histopathological abnormalities, including bronchial iron deposition and fibrosis [33-35].

Current concepts are that an excess of chelatable, or labile, iron derived from dysregulated cellular intake or export can cause hydroxyl radical generation in the presence of superoxide and hydrogen peroxide arising from the cell metabolism, with ensuing cell damage as hydroxyl radicals lead to oxidative damage with DNA breaks, lipid peroxidation and protein oxidation $[13,36]$. The H63D variant, which we found most frequently associated with pulmonary fibrosis in the current study patients, is associated with disease with very low penetrance, even when homozygous, when compared with the C282Y allele, which is the more commonly causative allele of the autosomal recessive type 1 hereditary haemochromatosis. However, both the $\mathrm{C} 282 \mathrm{Y}$ and the $\mathrm{H} 63 \mathrm{D}$ variants are associated with increased susceptibility to liver cirrhosis in alcohol liver disease and viral hepatitis [20,21]. Is the carriage of HFE gene variants a cause of dysregulated cellular iron levels in IPF? In a model of knock-in mouse, both C282Y and H63D HFE variants have been shown to increase cellular iron concentration, with H63D being less effective than C282Y [17], an observation that may explain the increased susceptibility to liver cirrhosis of HFE heterozygous individuals [21].

Environmental hazards, such as tobacco smoke, which are associated with accumulation of iron in the lower respiratory tract [22], have been indicated as IPF risk factors [23, 37], making it enticing to postulate tobacco as a cofactor of dysregulated iron accumulation. However, with the limitation of the small patient population and of the disproportionately small number of current smokers among the ever-smokers with IPF, we could not stratify HFE allelic variants by detailed smoking history or, in particular, by smoking cessation date [38]. We also could not stratify HFE allelic variants by age or sex, although the marked male prevalence in hereditary haemochromatosis [39] would entice one to hypothesise a role of sex-related iron accumulation in the marked age-related incidence shown in IPF in the UK as well as in central Italy [40, 41].

Nevertheless, these data add further evidence to BAL studies characterising alveolar macrophage/ neutrophilic alveolitis as a first step toward pulmonary fibrosis [4]. In addition to the already known accumulation of increased numbers of alveolar macrophages and of PMNs spontaneously releasing exaggerated amounts of oxygen radicals in the lower respiratory tract [4-6] in the presence of increased levels of lipid peroxidation activity and of reduced anti-oxidant capacity, the data demonstrate the presence of exaggerated levels of chelatable iron, i.e. the form of iron capable of catalysing the generation of oxygen 
radicals, probably the hydroxyl radical. What is more, this study shows that the H63D carriers display higher spontaneous iron-dependent ROS generation than patients who are homozygotes for the HFE wild type and that the latter still display higher unstimulated iron-dependent ROS generation than normal controls. However, the data strongly suggest that disordered iron regulation may play a central role in the pathogenesis of an oxidative stress-induced microscopic injury triggering epithelial cell damage and fibroblast proliferation. In this regard, the pathogenetic hypothesis stemming from this study is congruent with the findings of increased production of oxidants and oxidative damage in the IPF lower respiratory tract [4-6]. It is also reconcilable with the observation that telomere shortening, which has been found in a significant number of sporadic IPF patients without TERT or TERC mutations (mutations in genes encoding the protein component (TERT) and RNA component (TERC) of telomerase) [42], has been reported in populations of older individuals exposed to environmental hazards such as tobacco smoke, suggesting an independent role of oxidative stress in telomere abnormalities [43]. Large multicentre studies are needed to validate the finding that disordered iron homeostasis and exaggerated iron-dependent oxidant generation are associated with the carriage of HFE allelic variants in IPF. This study population was not sufficiently large for stratification analysis of the association of the genetic marker with pulmonary and cardiovascular abnormalities as well as disease severity and progressiveness. It is, however, enticing to hypothesise that persistent iron-mediated oxidative stress may be a likely disease-initiating or -modifying factor leading to, or amplifying, the epithelial cell damage and fibroblast proliferation occurring in the presence of the mild inflammatory reaction that characterises IPF.

\section{Acknowledgements}

The authors would like to thank R. du Bois (Imperial College, London, UK), L. Palombi, G. Novelli, M. Paci, M. Coletta (all University of Roma Tor Vergata, Rome, Italy) and A. Pietrangelo (University of Modena, Modena, Italy) for helpful discussions; L. Senis, P. Rogliani, C. Ciaprini, J. Ora, S. Portalone, A. Mari, A. Coppola, L. Matassi and A. Marocchi (all University Hospital Tor Vergata, Rome, Italy) for their invaluable help with clinical management of study patients; and S. Pantanella (Rome, Italy) for inspiring conversations.

Part of the data presented is the object of the patent application "Method for in vitro diagnosis of idiopathic pulmonary fibrosis" (PCT/IT2014/000214), submitted August 8, 2014.

\section{References}

1 Travis WD, Costabel U, Hansell DM, et al. An official American Thoracic Society/European Respiratory Society statement: Update of the international multidisciplinary classification of the idiopathic interstitial pneumonias. Am J Respir Crit Care Med 2013; 188: 733-748.

2 Raghu G, Collard HR, Egan JJ, et al. An official ATS/ERS/JRS/ALAT statement: idiopathic pulmonary fibrosis: evidence-based guidelines for diagnosis and management. Am J Respir Crit Care Med 2011; 183: 788-824.

3 Katzenstein AL, Mukhopadhyay S, Myers JL. Diagnosis of usual interstitial pneumonia and distinction from other fibrosing interstitial lung diseases. Hum Pathol 2008; 39: 1275-1294.

4 Crystal RG, Bitterman PB, Rennard SI, et al. Interstitial lung diseases of unknown cause. Disorders characterized by chronic inflammation of the lower respiratory tract (first of two parts). N Engl J Med 1984; 310: 154-166.

5 Strausz J, Müller-Quernheim J, Steppling H, et al. Oxygen radical production by alveolar inflammatory cells in idiopathic pulmonary fibrosis. Am Rev Respir Dis 1990; 141: 124-128.

6 Rahman I, Skwarska E, Henry M, et al. Systemic and pulmonary oxidative stress in idiopathic pulmonary fibrosis. Free Radic Biol Med 1999; 27: 60-68.

7 Burger RM, Peisach J, Horwitz SB. Activated bleomycin. A transient complex of drug, iron, and oxygen that degrades DNA. J Biol Chem 1981; 256: 11636-11644.

8 Chandler DB, Barton JC, Briggs DD 3rd, et al. Effect of iron deficiency on bleomycin-induced lung fibrosis in the hamster. Am Rev Respir Dis 1988; 137: 85-89.

9 Pinho RA, Silveira PC, Silva LA, et al. N-acetylcysteine and deferoxamine reduce pulmonary oxidative stress and inflammation in rats after coal dust exposure. Environ Res 2005; 99: 355-360.

10 Kim KH, Maldonado $\mathrm{F}$, Ryu $\mathrm{JH}$, et al. Iron deposition and increased alveolar septal capillary density in nonfibrotic lung tissue are associated with pulmonary hypertension in idiopathic pulmonary fibrosis. Respir Res 2010; 11: 37.

11 Puxeddu E, Comandini A, Cavalli F, et al. Iron laden macrophages in idiopathic pulmonary fibrosis: the telltale of occult alveolar hemorrhage? Pulm Pharmacol Ther 2014; 28: 35-40.

12 Bacon BR, Britton RS. The pathology of hepatic iron overload: a free radical-mediated process? Hepatology 1990; 11: $127-137$.

13 Feder JN, Penny DM, Irrinki A, et al. The hemochromatosis gene product complexes with the transferrin receptor and lowers its affinity for ligand binding. Proc Natl Acad Sci USA 1998; 95: 1472-1477.

14 Beutler E, Felitti VJ, Koziol JA, et al. Penetrance of 845G $\rightarrow$ A (C282Y) HFE hereditary haemochromatosis mutation in the USA. Lancet 2002; 359: 211-218.

15 Nemeth E, Tuttle MS, Powelson J, et al. Hepcidin regulates cellular iron efflux by binding to ferroportin and inducing its internalization. Science 2004; 306: 2090-2093.

16 Montosi G, Donovan A, Totaro A, et al. Autosomal-dominant hemochromatosis is associated with a mutation in the ferroportin (SLC11A3) gene. J Clin Invest 2001; 108: 619-623.

17 Tomatsu S, Orii KO, Fleming RE, et al. Contribution of the H63D mutation in HFE to murine hereditary hemochromatosis. Proc Natl Acad Sci USA 2003; 100: 15788-15793.

18 Njajou OT, Houwing-Duistermaat JJ, Osborne RH, et al. A population-based study of the effect of the HFE C282Y and H63D mutations on iron metabolism. Eur J Hum Genet 2003; 11: 225-231. 
19 Merryweather-Clarke AT, Pointon JJ, Shearman JD, et al. Global prevalence of putative haemochromatosis mutations. J Med Genet 1997; 34: 275-278.

20 Costa-Matos L, Batista P, Monteiro N, et al. Hfe mutations and iron overload in patients with alcoholic liver disease. Arq Gastroenterol 2013; 50: 35-41.

21 Erhardt A, Maschner-Olberg A, Mellenthin C, et al. HFE mutations and chronic hepatitis C: H63D and C282Y heterozygosity are independent risk factors for liver fibrosis and cirrhosis. J Hepatol 2003; 38: 335-342.

22 Thompson $\mathrm{AB}$, Bohling $\mathrm{T}$, Heires $\mathrm{A}$, et al. Lower respiratory tract iron burden is increased in association with cigarette smoking. J Lab Clin Med 1991; 117: 493-499.

23 Steele MP, Speer MC, Loyd JE, et al. Clinical and pathologic features of familial interstitial pneumonia. Am J Respir Crit Care Med 2005; 172: 1146-1152.

24 Meyer KC, Raghu G, Baughman RP, et al. An official American Thoracic Society clinical practice guideline: the clinical utility of bronchoalveolar lavage cellular analysis in interstitial lung disease. Am J Respir Crit Care Med 2012; 185: 1004-1014.

25 Golde DW, Drew WL, Klein HZ, et al. Occult pulmonary haemorrhage in leukaemia. Br Med J 1975; 2: 166-168.

26 Lazzarino G, Di Pierro D, Tavazzi B, et al. Simultaneous separation of malondialdehyde, ascorbic acid, and adenine nucleotide derivatives from biological samples by ion-pairing high-performance liquid chromatography. Anal Biochem 1991; 197: 191-196.

27 Esterbauer H, Lang J, Zadravec S, et al. Detection of malonaldehyde by high-performance liquid chromatography. Methods Enzymol 1984; 105: 319-328.

28 Bass DA, Parce JW, Dechatelet LR, et al. Flow cytometric studies of oxidative product formation by neutrophils: a graded response to membrane stimulation. J Immunol 1983; 130: 1910-1917.

29 Little J, Higgins JP, Ioannidis JP, et al. STrengthening the REporting of Genetic Association studies (STREGA) an extension of the STROBE statement. Eur J Clin Invest 2009; 39: 247-266.

30 Persson HL, Vainikka LK, Eriksson HB, et al. Lane-Hamilton syndrome: ferritin protects lung macrophages against iron and oxidation. Chest 2011; 139: 361-367.

31 Montani D, Price LC, Dorfmuller P, et al. Pulmonary veno-occlusive disease. Eur Respir J 2009; 33: 189-200.

32 Maldonado F, Parambil JG, Yi ES, et al. Haemosiderin-laden macrophages in the bronchoalveolar lavage fluid of patients with diffuse alveolar damage. Eur Respir J 2009; 33: 1361-1366.

33 Priftis KN, Anthracopoulos MB, Tsakanika C, et al. Quantification of siderophages in bronchoalveolar fluid in transfusional and primary pulmonary hemosiderosis. Pediatr Pulmonol 2006; 41: 972-977.

34 Khong PL, Chan GC, Lee SL, et al. Beta-thalassemia major: thin-section CT features and correlation with pulmonary function and iron overload. Radiology 2003; 229: 507-512.

35 Ooi GC, Khong PL, Lam WK, et al. Pulmonary iron overload in thalassemia major presenting as small airway disease. Acta Haematol 2002; 108: 43-46.

36 Ghoti H, Fibach E, Merkel D, et al. Changes in parameters of oxidative stress and free iron biomarkers during treatment with deferasirox in iron-overloaded patients with myelodysplastic syndromes. Haematologica 2010; 95: $1433-1434$.

37 Baumgartner KB, Samet JM, Coultas DB, et al. Occupational and environmental risk factors for idiopathic pulmonary fibrosis: a multicenter case-control study. Am J Epidemiol 2000; 152: 307-315.

38 Doll R, Peto R, Boreham J, et al. Mortality in relation to smoking: 50 years' observations on male British doctors. BMJ 2004; 328: 1519.

39 Floreani A, Rosa Rizzotto E, Basso D, et al. An open population screening study for HFE gene major mutations proves the low prevalence of C282Y mutation in Central Italy. Aliment Pharmacol Ther 2007; 26: 577-586.

40 Agabiti N, Porretta MA, Bauleo L, et al. Idiopathic pulmonary fibrosis (IPF) incidence and prevalence in Italy. Sarcoidosis Vasc Diffuse Lung Dis 2014; 31: 191-197.

41 Navaratnam V, Fleming KM, West J, et al. The rising incidence of idiopathic pulmonary fibrosis in the UK. Thorax 2011; 66: 462-467.

42 Cronkhite JT, Xing C, Raghu G, et al. Telomere shortening in familial and sporadic pulmonary fibrosis. Am J Respir Crit Care Med 2008; 178: 729-737.

43 Morlá M, Busquets X, Pons J, et al. Telomere shortening in smokers with and without COPD. Eur Respir J 2006; 27: $525-528$. 